\title{
Networking for infectious-disease emergencies in Europe
}

\author{
Giuseppe Ippolito, Carla Nisii and Maria R. Capobianchi
}

We read with great interest the editorial by Michael Head (Nature Reviews Microbiol. 6, $\left.328(2008)^{1}\right)$ who described the Infectious Disease Network that is in place in the United Kingdom. Because of the importance of promoting multi-disciplinary and multi-centre collaboration, we share in this Correspondence our experience as coordinators of European-Community-funded networks that operate in the area of preparedness and response to infectious-disease emergencies (IDEs).

We have been managing these networks since 2004, starting with a project that brought together infectious-disease clinicians who are expert in the management of highly infectious diseases (European Network for Highly Infectious Diseases (EURONHID)), followed by a Biosafety Level-4 (P4) laboratory network (European Network of P4 laboratories (Euronet-P4)) and a project that was entirely devoted to the interdisciplinary training of front-line health professionals (European Training for Infectious Disease Emergencies (ETIDE)).

Indeed, state-of-the-art medical care with high-level infection-control measures, rapid and accurate laboratory diagnosis performed in the appropriate containment facilities and inter-disciplinary training that is provided to all health-care workers involved (clinicians, nurses and first responders), together with appropriate risk evaluation, early warning systems and facility readiness, are the ingredients of a swift and effective response.

To this end, energy and effort needs to be directed towards increasing standardization of procedures and policies, given the diversity of cultural backgrounds and standards of care that exist today in the enlarged European Union, while providing assistance to countries that are not equipped with sophisticated facilities.

Our networks are good examples of successful cooperation, having brought together 19 European Union member states over the past few years; all participants are respected as experts in the field of highly infectious diseases and participate regularly in international meetings and crisis management exercises. Links are in place with all the pertinent European networks, including the WHO Global Outbreak Response Network (GOARN) and the Global Health Security Action Group Laboratory Network (GHSAG-LN).

Advances over the past 4 years include: the assessment of isolation capabilities in Europe; the development of expert agreements on infection-control procedures, the management of invasive procedures in patients who are highly infectious and technical specifications for high-level isolation units; the collection of biosafety and training procedures that have been adopted in European P4 laboratories; the collection, sharing and evaluation of diagnostic methods for P4 agents; the development of core curriculums for health-care workers who deal with IDEs; and the introduction of 1-week courses on the management of IDEs.

Further details can be found on the three project web sites: EUNiD (www.eunid.eu), EURONETP4 (www.euronetp4.com) and ETIDE (www.etide.eu).

1. Head, M., Hayward, A. \& Johnson, A. Networking for infectious disease. Nature Rev. Microbiol. 6, 328 (2008)

Giuseppe Ippolito, Carla Nisii, and Maria R. Capobianchi are at the National Institute for Infectious Diseases, INMI L. Spallanzani, Rome 00149, Italy. Correspondence to G.I. and M.R.C. e-mail: ippolito@inmi.it; capobianchi@inmi.it 